\title{
Overcoming immune-cell unresponsiveness in cancer
}

\author{
Guenther Lametschwandtner ${ }^{1 *}$, Monika Ermann², Ina Sternberger ${ }^{3}$, Thomas Hesterkamp $^{3}$, Isabella Haslinger ${ }^{1}$, \\ Hannes Mühleisen ${ }^{1}$, Manfred Schuster ${ }^{1}$, Hans Loibner ${ }^{1}$ \\ From Society for Immunotherapy of Cancer 28th Annual Meeting \\ National Harbor, MD, USA. 8-10 November 2013
}

Despite significant recent progress, cancer immunotherapy efficacies are still limited, since tumors can downmodulate and escape the immune system by various mechanisms. To overcome these limitations, we aimed to identify low molecular weight molecules that can enhance $\mathrm{T}$ cell reactivity in the context of otherwise insufficient or suppressive stimulation. In an HTS campaign, 80000 maximal diverse compounds were screened on anti-CD3/CD28 stimulated human PBMCs using enhancement of IL2 production as readout. The confirmed hit list contained 3 chemically independent series, which enhanced the production of inflammatory cytokines, especially IL- 2 , IFN- $\gamma$ and TNF- $\alpha$, from TCRstimulated $\mathrm{T}$ cells, while lacking any agonist activity on unstimulated $\mathrm{T}$ cells. The compound-mediated enhancement of TCR-stimulation was also observed on the level of $\mathrm{T}$ cell activation markers like CD25, CD69, CD71 and CD40L. In line with these observations, compounds of these series enhanced the in vitro anti-tumor responsiveness of PBMCs against tumor cell lines, originating from melanoma (M21), neuroblastoma (LAN) and leukemia (K562). For MHC-positive tumor cells, refined analysis demonstrated that compounds enhanced CD8 T cell activation, resulting in selective tumor cell killing while compounds in the absence of immune cells did not affect tumor cell viability. In contrast, the PBMCresponse against $\mathrm{K} 562$ cells, which is a commonly used NK cell target due to the lacking MHC-expression, was mainly mediated by NK cells. Moreover, compounds also enhanced IL-2 mediated NK cell proliferation and cytokine (IFN- $\gamma$, TNF- $\alpha$ ) and effector molecule (Granzyme B, Perforine) secretion. The activities of these compounds were further confirmed in an antigen-specific ex vivo $T$ cell stimulation assay of human PBMCs with Tetanus toxoid, which resulted in enhanced proliferation of $\mathrm{T}$ cells. In addition, enhanced IL-2 secretion by activated T cells led to consequently increased NK cell activation and proliferation in antigen-stimulated stimulated PBMCs. To enable future assessment of compound activities in murine tumor models in vivo, we have verified that compounds of all 3 series enhanced $\mathrm{T}$ cell activation and cytokine production of TCR-stimulated murine T cells, while they had no effect on unstimulated $\mathrm{T}$ cells. Together, we show here the identification of a novel class of low molecular weight compounds with drug-like properties that selectively enhance activation of T and NK cells in the context of antigen-specific and anti-tumor immunity with high potential for development of improved tumor immunotherapeutics.

\section{Authors' details \\ ${ }^{1}$ Apeiron Biologics, Vienna, Austria. ${ }^{2}$ Evotec, Abingdon, UK. ${ }^{3}$ Evotec, Hamburg, Germany.}

Published: 7 November 2013

doi:10.1186/2051-1426-1-S1-P261

Cite this article as: Lametschwandtner et al:: Overcoming immune-cell unresponsiveness in cancer. Journal for ImmunoTherapy of Cancer 2013 1(Suppl 1):P261.

${ }^{1}$ Apeiron Biologics, Vienna, Austria

Full list of author information is available at the end of the article

(c) 2013 Lametschwandtner et al; licensee BioMed Central Ltd. This is an Open Access article distributed under the terms of the Creative 